\title{
The Relationship between Spiritual and Moral Intelligence with the Meaning of Life among the Volunteers of the Emergent Reaction
}

\author{
Sadigheh Golipour*, Sara Haghighat, Davood Manavipour
}

Department of Psychology, Faculty of Psychology and Educational Sciences, Garmsar Branch, Islamic Azad University, Garmsar, Iran.

\section{A BSTRACT}

Introduction: The volunteers of the emergent reaction are the groups of people who play a vital role after natural disasters and events. The meaning of life can have the vital role in the stability, internal motivation, and the achievement of these people during their rescue activities. The purpose of this research was to investigate the relationship between spiritual intelligence and moral intelligence as important variables in the meaning of life among a group of Iranian volunteers of the emergent reaction in 2013-2014. Materials and Methods: The research population includes all the volunteers of emergency reaction in Shahr-e Rey, Tehran, Iran. The research sample included 184 men and women who were selected by multistage random sampling method. To measure the variables, spiritual intelligence, moral intelligence, and the meaning of life questionnaires were used. Pearson correlation, multivariate regressions analyses, and T-tests were used for statistical analysis of the data. Results: The results revealed a significant positive relationship between the meaning of life and spiritual intelligence as well as the meaning of life and moral intelligence. The results were exposed that spiritual intelligence explains 5.6 percent of the observed difference in the meaning of life among men and 18.6 among women. In addition, moral intelligence explains the variation of life meaning among $13.2 \%$ of men and $6.8 \%$ of women. There was no significant relationship between spiritual intelligence, moral intelligence, and meaning of life between men and women. Conclusion: These data suggest the crucial role of spiritual and moral intelligence with the meaning of life in the volunteers of the emergent reaction.

\section{Key words:}

1. Intelligence

2. Morals

3. Life

4. Volunteers

* Corresponding Author: Sadigheh Golipour

E-mail: Sadigheh.golipour@gmail.com 


\title{
رابطه هوش معنوى و هوش اخلاقى با معناى زندكى در داوطلبين واكنش اضطرارى
}

\author{
صديقه گللى يور"، سارا حقيقت، داوود معنوى يور
}

كروه روانشناسى، دانشكده روانشناسى و علوم تربيتى، واحد گرمسار، دانشكاه آزاد اسلامى، كرمسار، ايران.

كليد وازهها:

1

r.

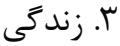

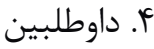

" نويسنده مسئول: صديقه گلى يور

آدرس الكترونيكى: Sadigheh.golipour@gmail.com
مقدمهـ: داوطلبين واكنش اضطرارى كروههاى مردمى هستند كه پِ از وقوع بلايا و حوادث طبيعى نقش حياتى بر عهده

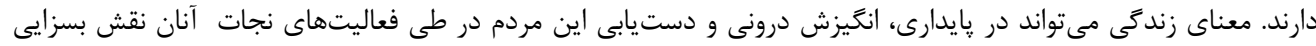

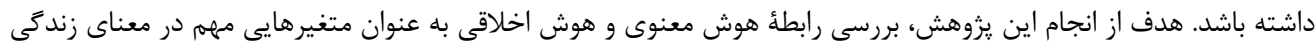

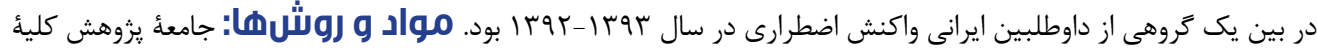

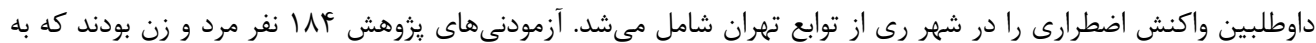

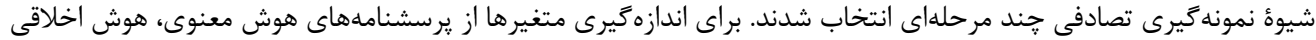

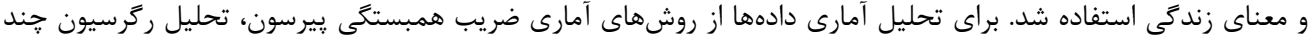

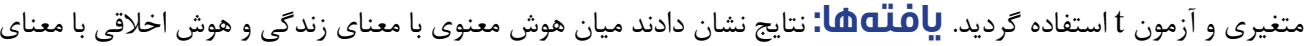

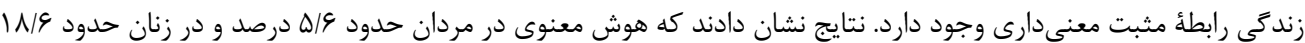

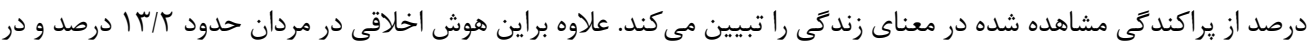

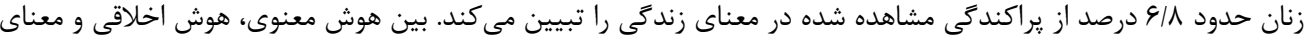

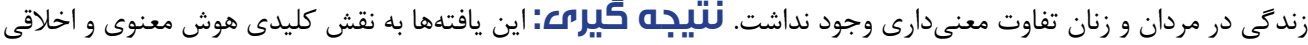

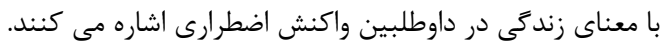


كروهى، افزايش بهرهورى، تصميم گيرى بر مبناى اصول اخلاقى إنى

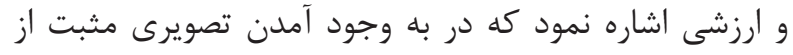

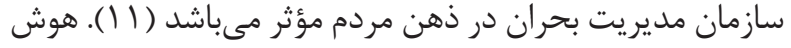

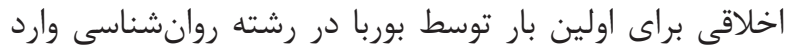

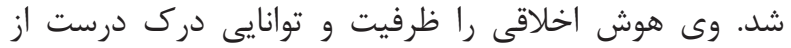

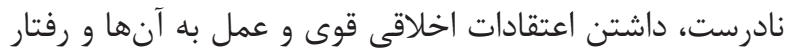

در جهت صحيح و درست تعريف كرده است (Y) (I)

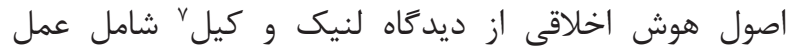

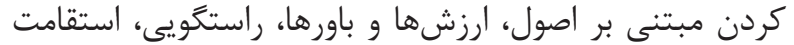

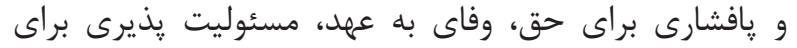

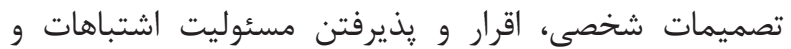

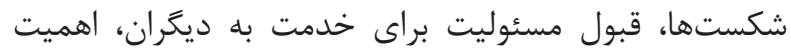

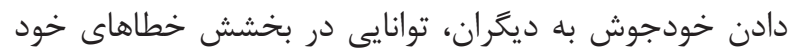

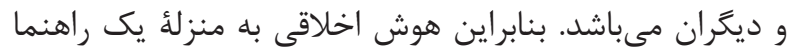

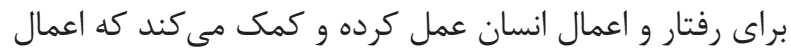

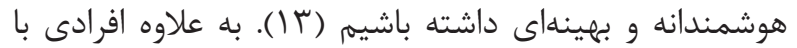

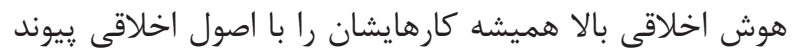

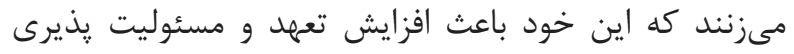

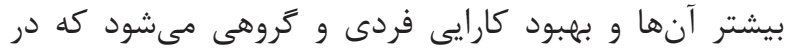

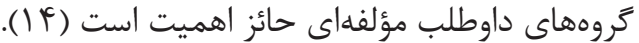

همجنين رابطؤ هوش اخلاقى با خود كنترلى رفتار (Q) (1)،

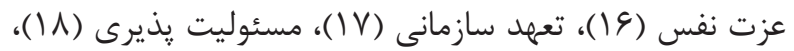

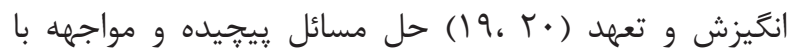

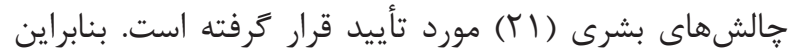

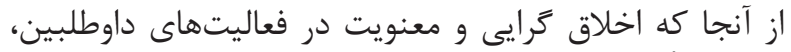

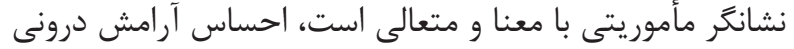

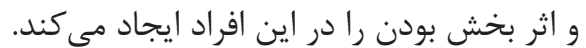

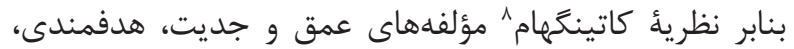

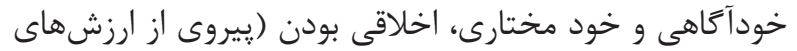

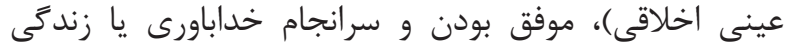

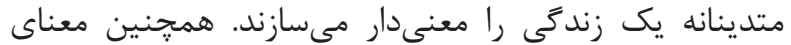

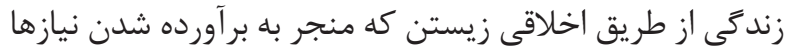

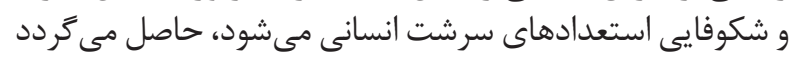

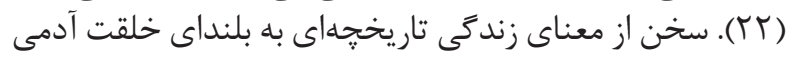

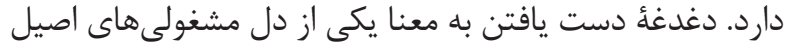

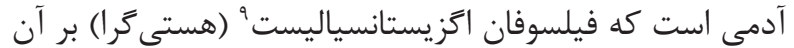

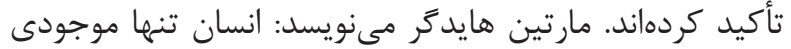
است كه وجودش براى او مسأله است (بارئ.

يكى از معروفترين تعاريف معناى زندگى، توسط فرانكل در سال

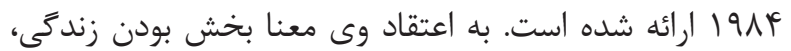

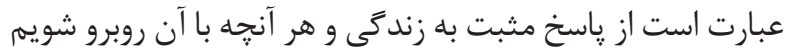

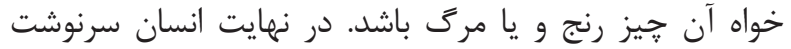

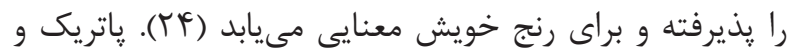

${ }^{1}$ Emergency response volunteers

${ }^{2}$ Stevens

${ }^{3}$ Emmons

${ }^{4}$ Zohar and Marshal

${ }^{5}$ Vaughan
مقدمه

كروههاى داوطلب واكنش اضطرارى' محله يا دوام، كروههاى

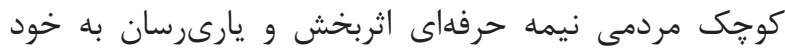

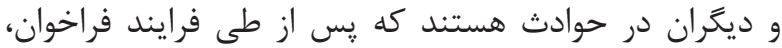

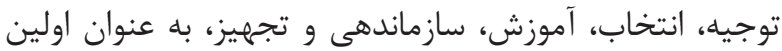

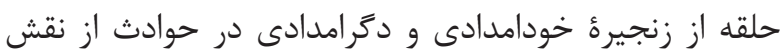

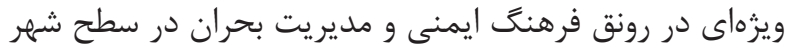

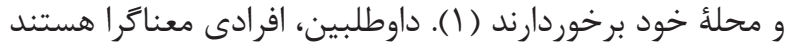

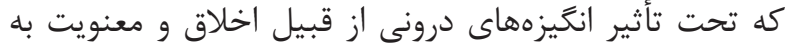

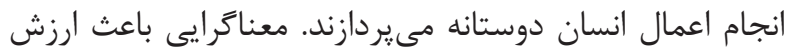

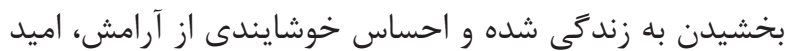

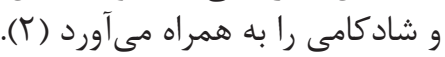

يكى از عوامل مؤثر در كرايش داوطلبين اضطرارى به اين

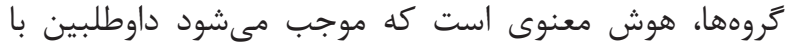

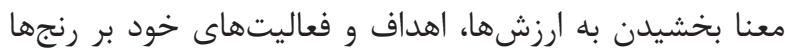

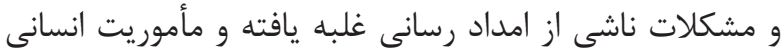

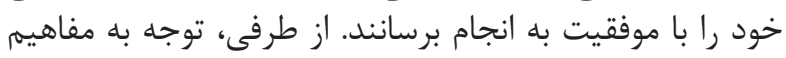

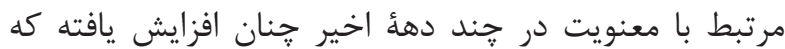

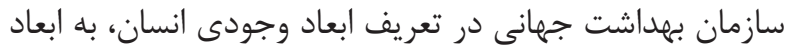

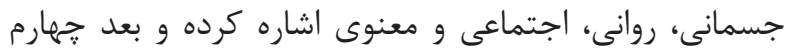

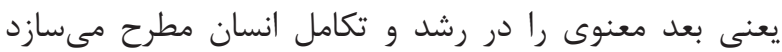

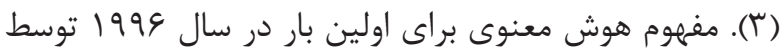

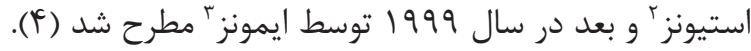

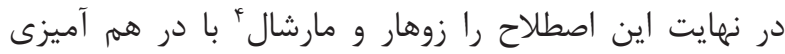

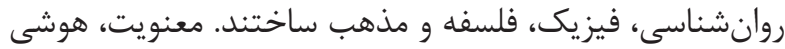

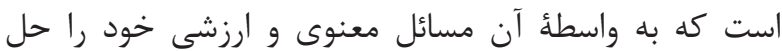

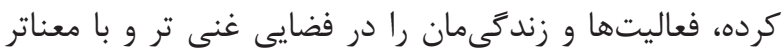

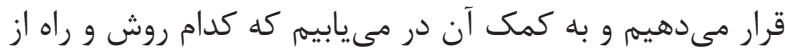

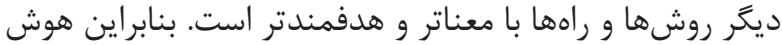

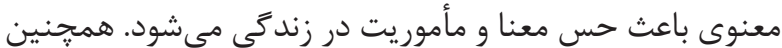

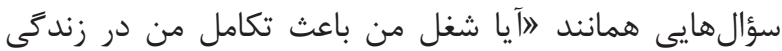

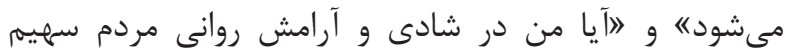

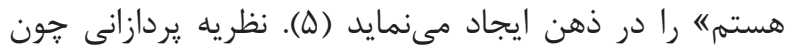

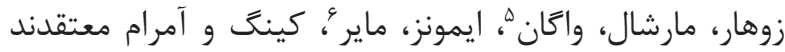

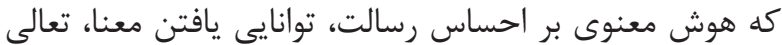

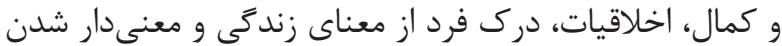

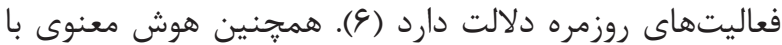

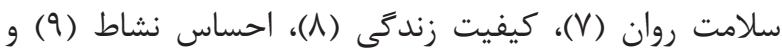

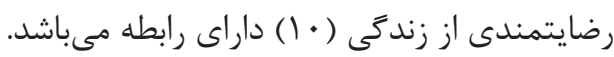

از سويى، هوش اخلاقى يكى ديكر از ابعاد هوش است كه

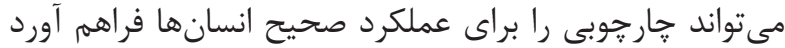

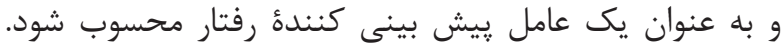

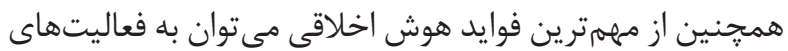

\footnotetext{
${ }^{6}$ Meyer

${ }^{7}$ Lennik and Kiel

${ }^{8}$ Cottingham

${ }^{9}$ Existentialist
} 
اضطرارى زن و مرد به صورت تصادفى انتخاب شدند كه در

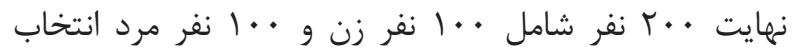

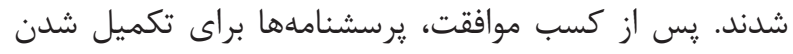

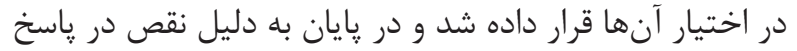

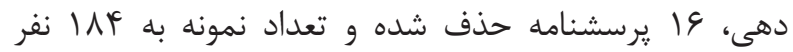

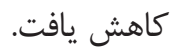

در اين يزوهش براى جمع آورى اطلاعات آزمودنى ها از يرسشنامئ

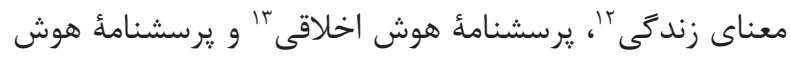

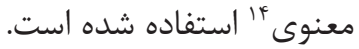

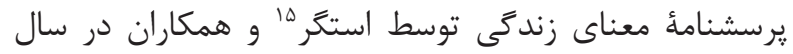

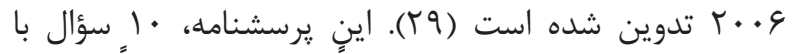

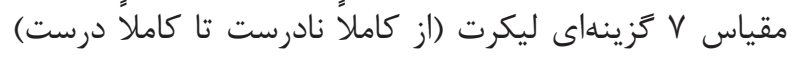

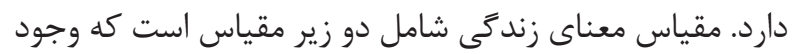

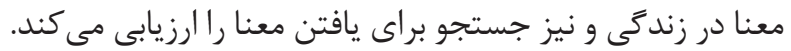

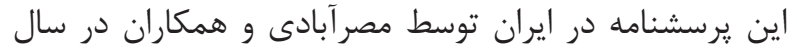

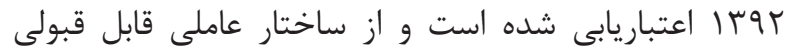

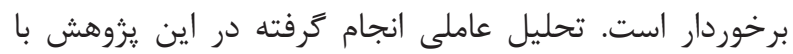

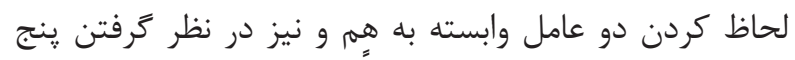

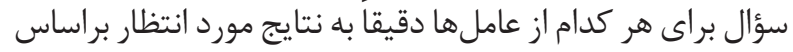

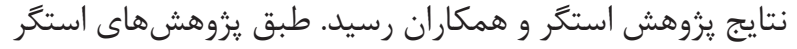

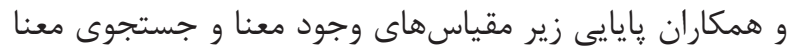

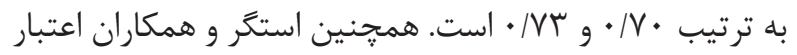

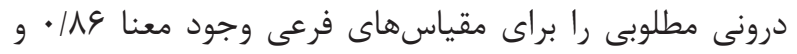

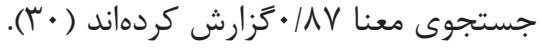

تيرسشنامٔ هوش اخلاقى توسط لنيك و كيل در سال هـ •

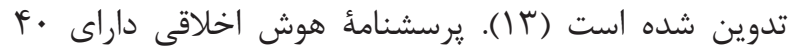

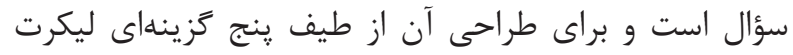

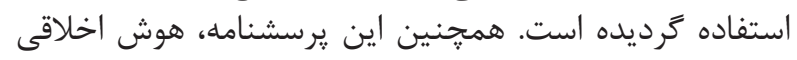

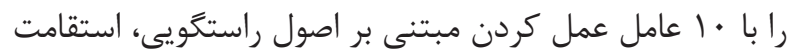

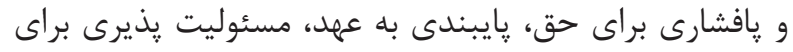

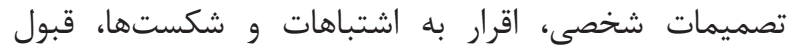

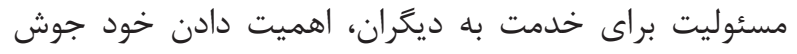

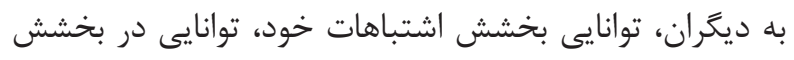

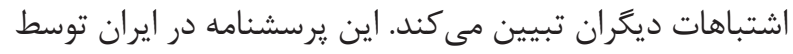

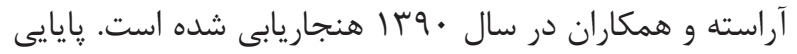

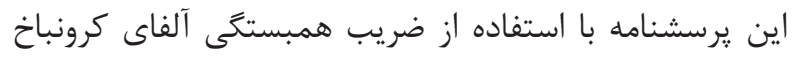

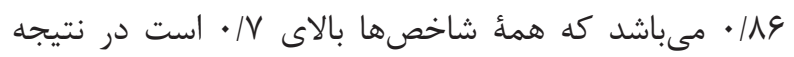

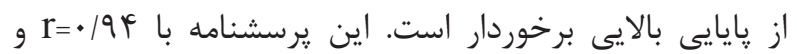

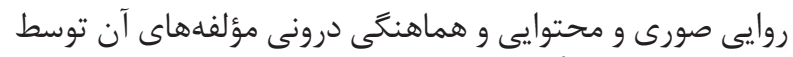

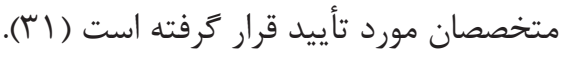

يرسشنامة هوش معنوى در سال 11199 توسط شاه حسينى و

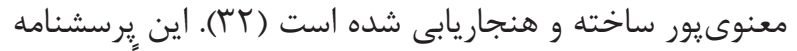

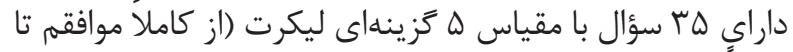

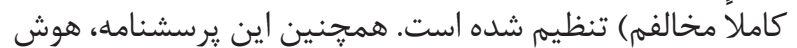

${ }^{10}$ Joseph and Lakshmi

${ }^{11}$ McMahan and Renken

${ }^{12}$ Meaning of life questionnaire
اندرسون در سال 1919 با يزوهشى نشان دادند كه وجود معنا

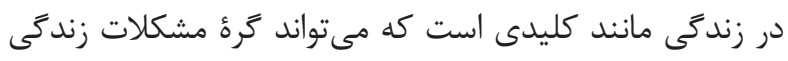

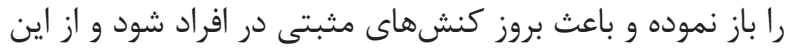

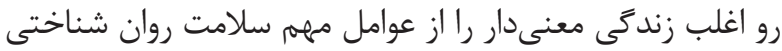

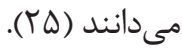

در راستاى مطالب فوق، تحقيقاتى نيز انجام شده است؛ نادى و

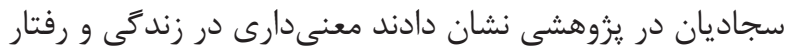

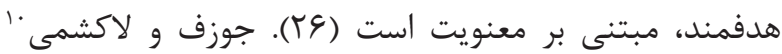

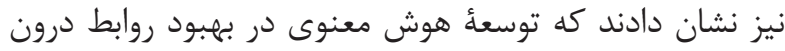

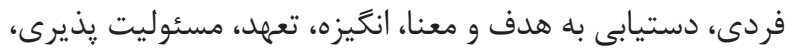

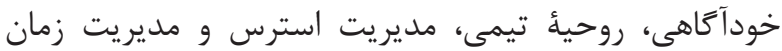

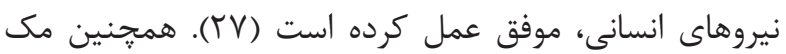

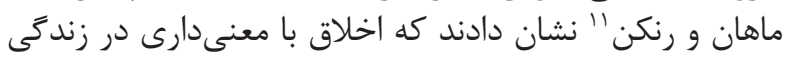

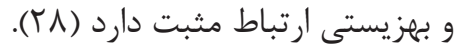

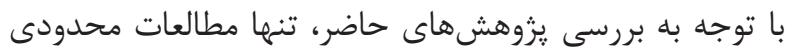

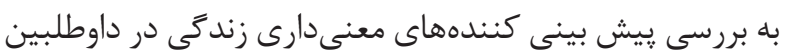

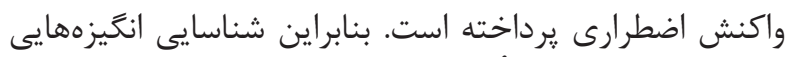

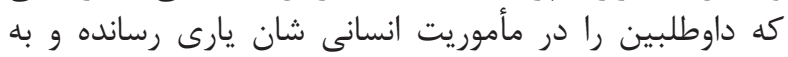

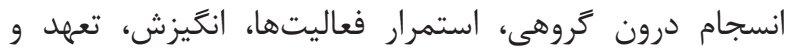

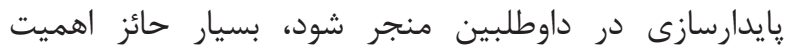

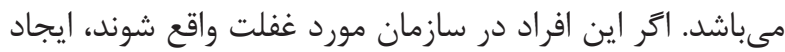

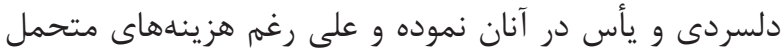

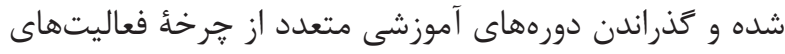

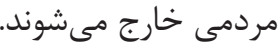

لذا، مسئلة اصلى يزوهش حاضر اين است كه آيا بين هوش

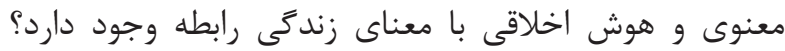

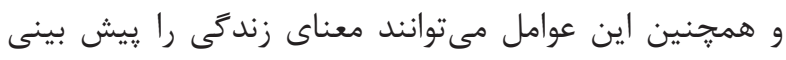

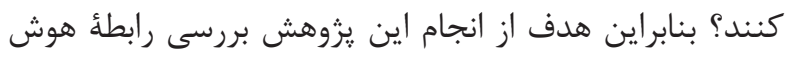

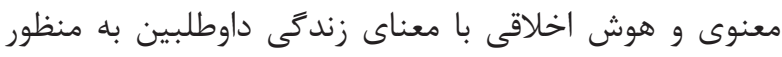

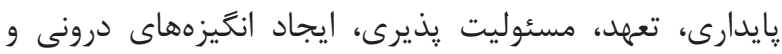
استفاده بهينه از نيروهاى مردمى در شرايط بحئ بحرانى بوده است.

\section{مواد و روشها}

يثوهش حاضر از نوع يزوهشهاى توصيفى و در جارجوب

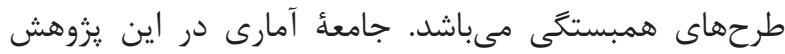

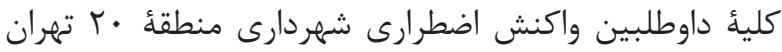

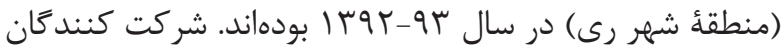

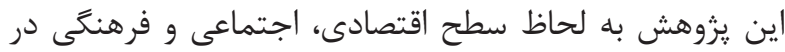

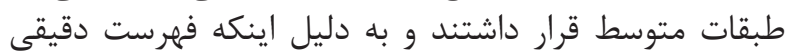

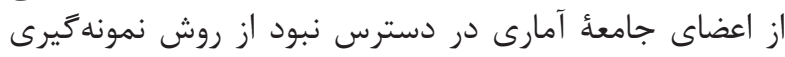

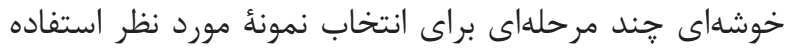

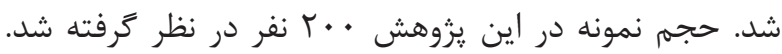

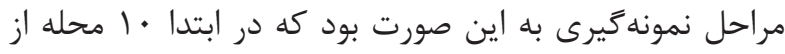

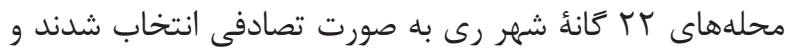

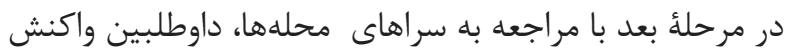

\footnotetext{
${ }^{13}$ Moral intelligence questionnaire

${ }^{14}$ Spiritual intelligence questionnaire

${ }^{15}$ Steger
} 


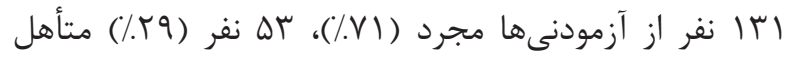

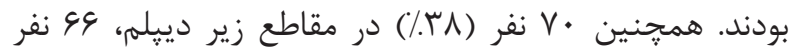

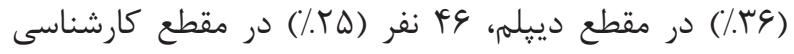

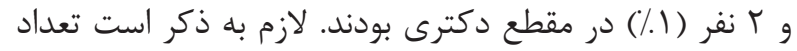

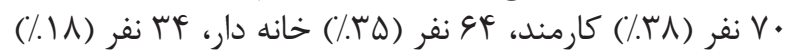

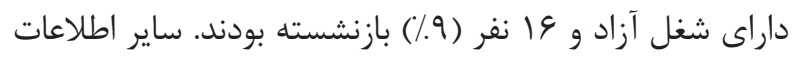

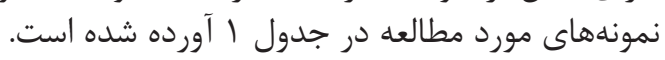

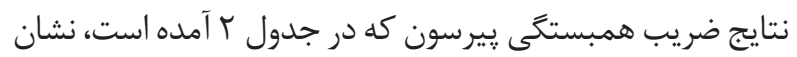

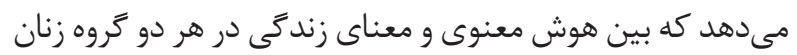

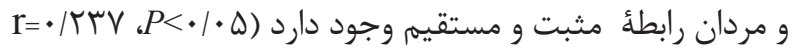

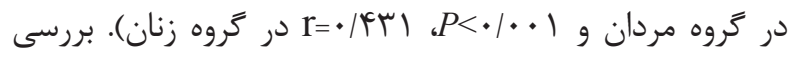

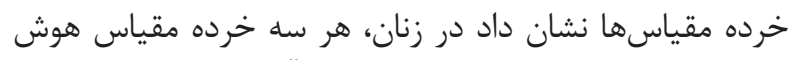

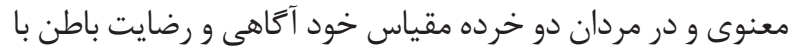

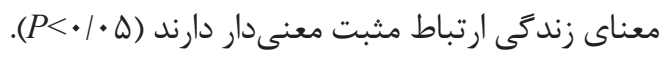

در ادامه براى بررسى اين كه هوش معنى معنوى خند دئد درصد از

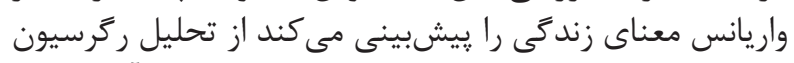

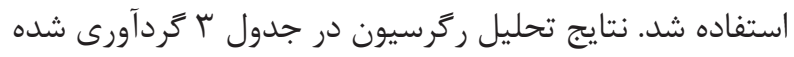

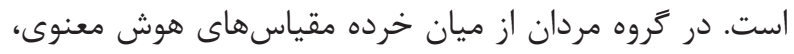

معنوى را با ب عامل اخلاق مدارى، خودآكاهى و رضايت باطن

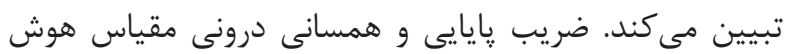

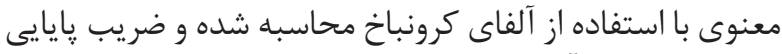

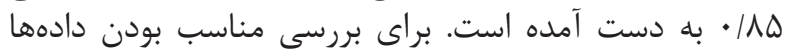

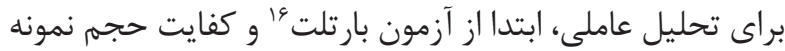

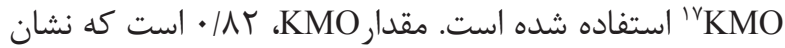

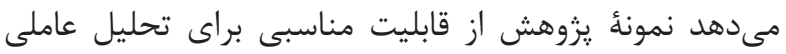

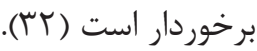

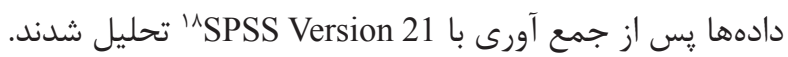

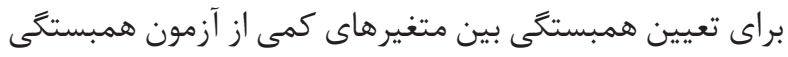

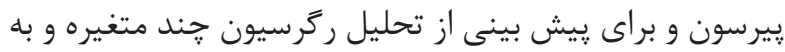

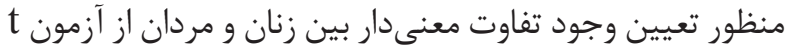

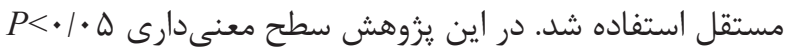
در نظر گرفته شد.

بافتهها

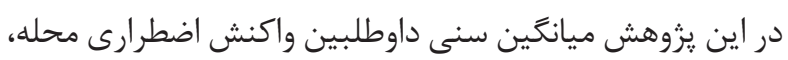

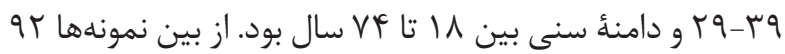

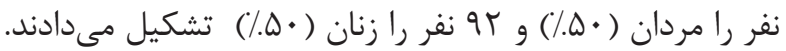

جدول ا- شاخصهاى توصيفى متغيرهاى يزوهش.

\begin{tabular}{|c|c|c|c|c|}
\hline \multicolumn{2}{|c|}{ زن } & \multicolumn{2}{|c|}{ مرد } & \multirow{2}{*}{ متغير } \\
\hline انحراف معيار & ميانغين & انحراف معيار & ميانغين & \\
\hline$V / \Delta \& V$ & $\Delta \wedge / F \& V$ & $9 / 9 r$ & $\Delta \Lambda / T Y \Lambda$ & معناى زندَّى \\
\hline I F/VAr & $\mid F q / 9 \vee \wedge$ & $\mid \Delta / \cdot 19$ & IFN/DTY & هوش معنوى \\
\hline س & $\mid \& \Psi / \backslash \wedge F$ & $1 \Delta / \wedge \Delta q$ & $\mid \Delta q / \cdot 1$ & هوشى اخلاقى \\
\hline
\end{tabular}

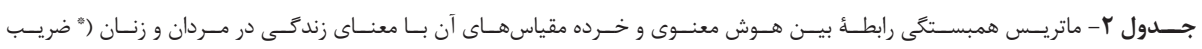

\begin{tabular}{|c|c|c|c|}
\hline معناى زندكى (زن) & معناى زندكى (مرد) & & 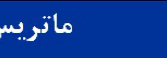 \\
\hline$\cdot|A T|$ & . /THV & $\mathrm{R}$ & \multirow{3}{*}{ هوش معنوى } \\
\hline$\cdot|\cdots|$ & /. & Sig. & \\
\hline 95 & qr & $\mathrm{N}$ & \\
\hline . /4TG: & $\cdot / 119$ & $\mathrm{R}$ & \multirow{3}{*}{ اخلاق مدارى } \\
\hline$\cdot / \cdots 1$ & $\cdot / \cdot \sqrt{ } 9$ & Sig. & \\
\hline 95 & $9 r$ & $\mathrm{~N}$ & \\
\hline ./TYG* & ./TH": & $\mathrm{R}$ & \multirow{3}{*}{ خود آكاهى } \\
\hline$\cdot / \cdot 4$ &.$/ . t F$ & Sig. & \\
\hline 95 & $9 r$ & $\mathrm{~N}$ & \\
\hline$\cdot / r \Delta \Lambda^{*}$ & $\cdot /$ ४ & $\mathrm{R}$ & \multirow{3}{*}{ رضايت باطن } \\
\hline$\cdot / \cdot 11$ & $\cdot / \cdots+$ & Sig. & \\
\hline 95 & 95 & $\mathrm{~N}$ & \\
\hline
\end{tabular}

${ }^{16}$ Bartlett's test

${ }^{17}$ Kaiser Mayer Olkin

${ }^{18}$ Statistical package for social science (SPSS) 
جدول r- نتايج تحليل ركرسيون بين هوش معنوى و خرده مقياسهاى آن با معناى زندكى در مردان و زنان.

\begin{tabular}{|c|c|c|c|c|c|c|c|}
\hline Sig. & $\mathbf{T}$ & $\beta$ & $\mathbf{F}$ & $\mathbf{R}^{2}$ & $\mathbf{R}$ & مدل & جنسيت \\
\hline$\cdot / \cdots 1$ & $0 / 999$ & \multirow{2}{*}{ •/THV } & \multirow{2}{*}{ } & \multirow{2}{*}{$\cdot \mid \cdot \Delta \xi$} & \multirow{2}{*}{$\cdot / T H V$} & مقدار ثابت & \multirow[b]{2}{*}{ مرد } \\
\hline H & $r / 4 \cdot q$ & & & & & هوش معنوى & \\
\hline$\cdot / \cdots 1$ & $r / 4 \Delta \Lambda$ & \multirow{2}{*}{. } & \multirow{2}{*}{$r \cdot / \Delta r$} & \multirow{2}{*}{$\cdot / 119$} & \multirow{2}{*}{. $/ F T \mid$} & مقدار ثابت & \multirow{2}{*}{ زن } \\
\hline $.1 \cdots 1$ & F/DTH & & & & & هوش معنوى & \\
\hline$\cdot / \cdots 1$ & N/TGF & \multirow{2}{*}{.$/ 199$} & \multirow{2}{*}{ N/AFV } & \multirow{2}{*}{.$/ .9$} & \multirow{2}{*}{$\cdot / 1999$} & مقدار ثابت & \multirow[b]{2}{*}{ مرد } \\
\hline$\cdot / \cdot f$ & T/qVF & & & & & رضايت باطن & \\
\hline$\cdot / \cdots 1$ & $F / .91$ & \multirow{2}{*}{. } & \multirow{2}{*}{$T Y / .9$} & \multirow{2}{*}{.119.} & \multirow{2}{*}{. } & مقدار ثابت & \multirow[b]{2}{*}{ زن } \\
\hline $1 \cdots 1$ & $f / \Delta q$. & & & & & اخلاق مدارى & \\
\hline
\end{tabular}

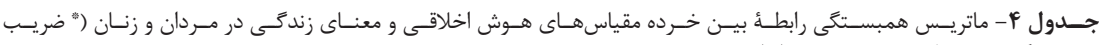

\begin{tabular}{|c|c|c|c|}
\hline معناى زندكى (زن) & معناى زفنىى (مره) & & ماتريس همبستكى \\
\hline$+(T Q)^{\prime}=$ & +/TGf= & $\mathrm{R}$ & \multirow{3}{*}{ هوش اخحالقى } \\
\hline$+1+14$ & $+1+\cdots+$ & Sig. & \\
\hline ar & 94 & $\mathrm{~N}$ & \\
\hline - $/$ thfF= & $\cdot / 4 T A^{2}$ & $\mathrm{R}$ & \multirow{3}{*}{ عمل كردن عيتنى بر اصول } \\
\hline$+1+1$ & $+1+\cdots$ & Sig. & \\
\hline ar & at & $\mathrm{N}$ & \\
\hline$+11 \mathrm{rr}$ & A/triz & $\mathrm{R}$ & \multirow{3}{*}{ راستكويع } \\
\hline .1194 & $+1+\cdots$ & Sig. & \\
\hline at & at & $\mathrm{N}$ & \\
\hline - $/ 4 G 4=$ & $+/ 4 \lambda^{*}=$ & $\mathrm{R}$ & \multirow{3}{*}{ الستقاعت و يافشارى براى حق } \\
\hline$+1+11$ & $+1+\cdots+1$ & Sig. & \\
\hline 94 & ar & $\mathrm{N}$ & \\
\hline$+/ T \wedge \Delta^{\circ}=$ & $+(f) 1=$ & $\mathrm{R}$ & \multirow{3}{*}{ باليتلىى به عبل } \\
\hline$+1+9$ & $+1+\cdots+1$ & Sig. & \\
\hline 9т & वर & $\mathrm{N}$ & \\
\hline$\cdot / T f \lambda^{\prime}=$ & $+/ T \wedge \gamma^{2}$ & $\mathrm{R}$ & \multirow{3}{*}{ عستوليت بتيرى براى تصعيعات } \\
\hline$+1+1 \mathrm{Y}$ & $+1+9$ & Sig. & \\
\hline at & at & $\mathrm{N}$ & \\
\hline$+119 \mathrm{Y}$ & +1199 & $\mathrm{R}$ & \multirow{3}{*}{ اقرار بـ شكستها و الشتباهات } \\
\hline+1111 & $+11+1$ & Sig. & \\
\hline at & ar & $\mathrm{N}$ & \\
\hline$+/ T \cdot f$ & $+/ 49+=$ & $\mathrm{R}$ & \multirow{3}{*}{ قيول عسنوليت براى خلعبك } \\
\hline$+1+\Delta r$ & $+1+14$ & Sig. & \\
\hline at & ar & $\mathrm{N}$ & \\
\hline$+1+99$ & ./TTO $=$ & $R$ & \multirow{3}{*}{ اهعيت دادن خوه حوش بـ ديكران } \\
\hline.$/ 149$. & $+1+t A$ & Sig. & \\
\hline at & at & $\mathrm{N}$ & \\
\hline.$/ 4 T A_{0}$ & $\cdot 11 \cdot \Delta$ & $\mathrm{R}$ & \multirow{3}{*}{ توالفايع بخشش اشتباهات خود } \\
\hline$+1+r$ & ./Tt. & Sig. & \\
\hline at & at & $\mathrm{N}$ & \\
\hline.$/ 1 Y G$ & +1191 & $\mathrm{R}$ & \multirow{3}{*}{ توالايع در بخشش اشئران } \\
\hline.$/ 4 \pi$ & HITf & Sig. & \\
\hline ar & at & $\mathrm{N}$ & \\
\hline
\end{tabular}


كنار كذاشته شدند.

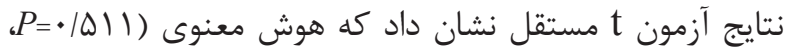

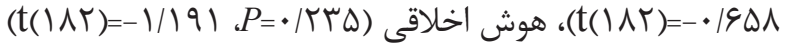
و معناى زند زنان و مردان رابطة معنى دارى دارند.

$$
\text { بحث و نتيجه تَيرى }
$$

نتايج مطالعهُ حاضر نشان داد بين هوش معنوى و هوش اخلاقى

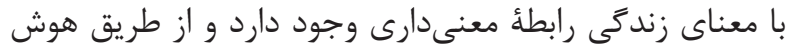

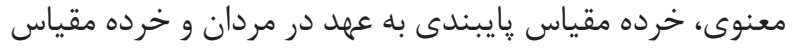

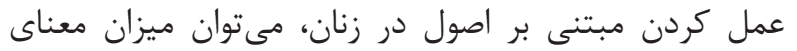

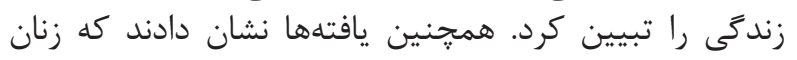

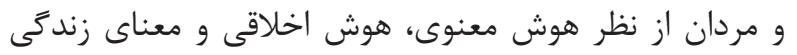

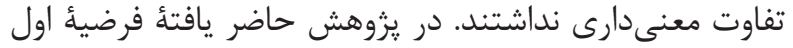

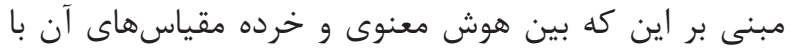

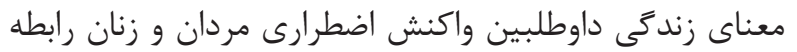

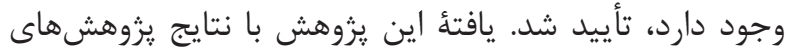

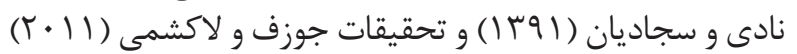

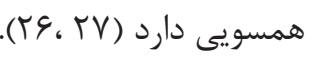

در تبيين اين فرضيه مىتوان كفت كه آمرام (9 + . ؟) معتقد

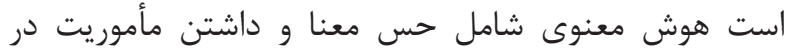

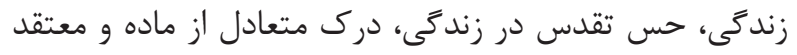

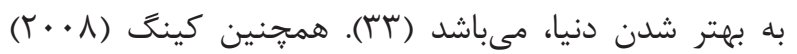

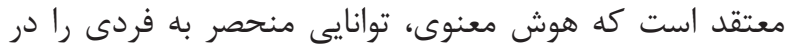

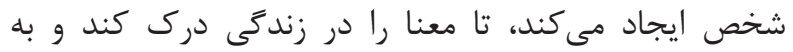

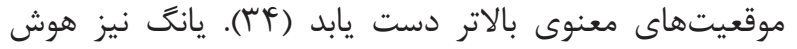

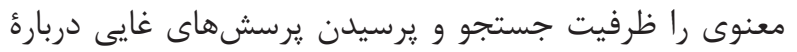

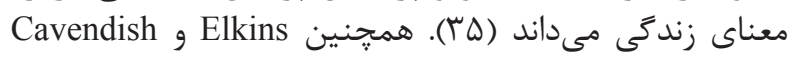

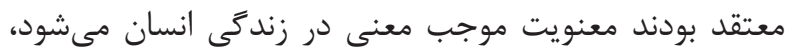

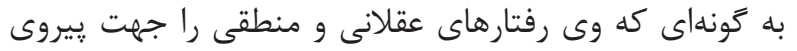

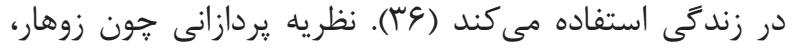

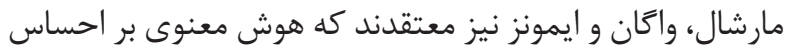

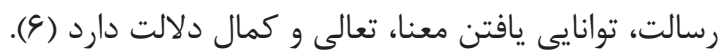

رضايت باطن در گام اول وارد معادله شده و نتايج نشان داد كه

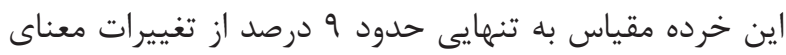

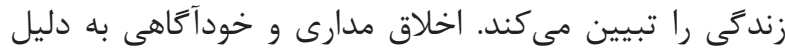

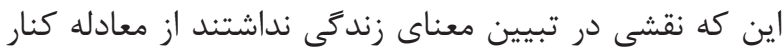

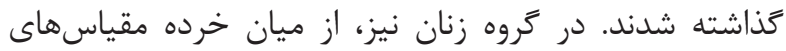
هوش معنوى، اخلاق مدارى در گَام اول وارد معادله

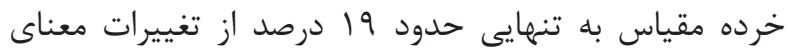

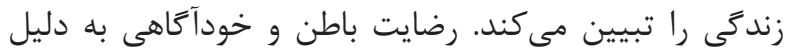

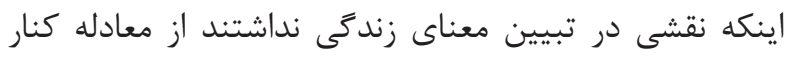

كذاشته شدند.

نتايج ضريب همبستگى ييرسون كه در جدول \& ا آورده شده

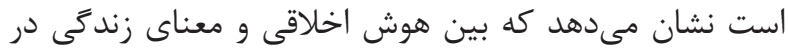

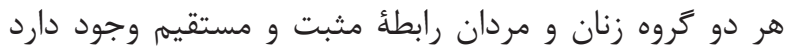

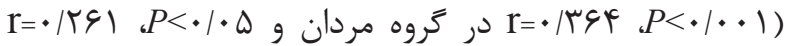

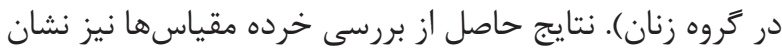

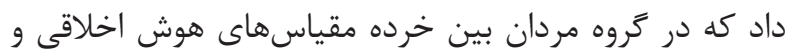

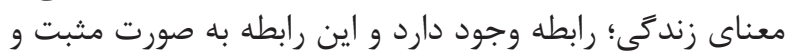

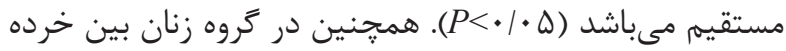

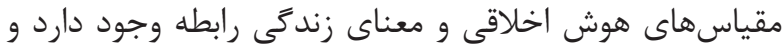

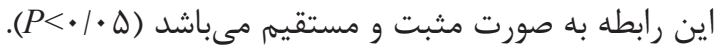

در ادامه براى بررسى اينكه خرده مقياسهاى هوش اخلاقى

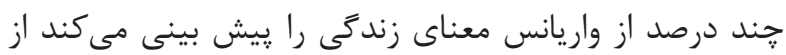

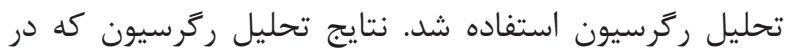

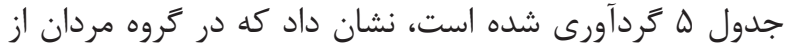

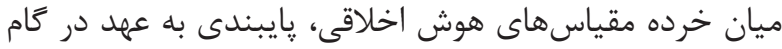

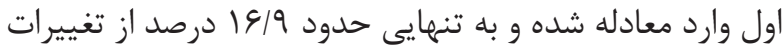

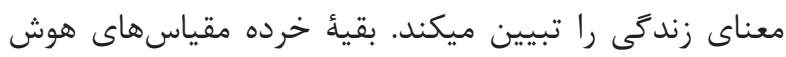

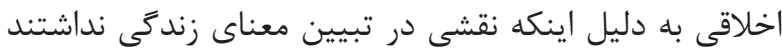

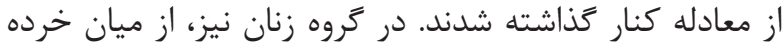

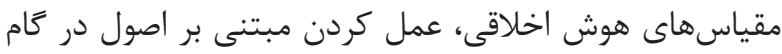

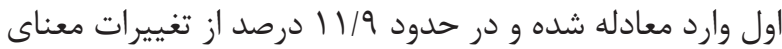

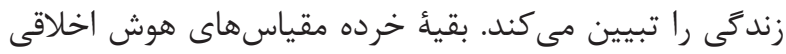

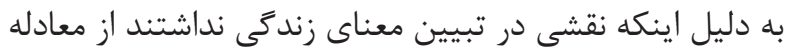

\begin{tabular}{|c|c|c|c|c|c|c|c|}
\hline Sig. & $\mathbf{T}$ & $\beta$ & F & $\mathbf{R}^{2}$ & $\mathbf{R}$ & مدل & جنسيت \\
\hline$\cdot / \cdots 1$ & $F / \Lambda I V$ & \multirow{2}{*}{. MGY } & \multirow{2}{*}{$1 \pi / V r$} & \multirow{2}{*}{. /MT } & \multirow{2}{*}{. TGY } & مقدار ثابت & \multirow{2}{*}{ مرد } \\
\hline$\cdot|\cdots|$ & $r / V \cdot V$ & & & & & هوش اخلاقى & \\
\hline$\cdot 1 \cdot \cdot 1$ & $11 / \cdot 48$ & \multirow{2}{*}{$\cdot|T \&|$} & \multirow{2}{*}{$\varepsilon / \Delta V F$} & \multirow{2}{*}{$.1 .9 \wedge$} & \multirow{2}{*}{$\cdot|T \&|$} & مقدار ثابت & \multirow{2}{*}{ زن } \\
\hline $.1 \cdot 1 \mathrm{~T}$ & T/DGF & & & & & هوش اخلاقى & \\
\hline$\cdot \mid \cdots \cdot$ & V/TTF & \multirow{2}{*}{$\cdot|f| \mid$} & \multirow{2}{*}{$\mid N / T \Lambda$} & \multirow{2}{*}{.1199} & \multirow{2}{*}{$\cdot|f| \mid$} & مقدار ثابت & \multirow{2}{*}{ مرد } \\
\hline$\cdot \mid \cdots \cdot$ & f/TVE & & & & & ֶايبندى بـ عهد & \\
\hline$\cdot \mid \cdots \cdot$ & G/AF & \multirow[b]{2}{*}{. MFF } & \multirow[b]{2}{*}{$1 \pi / 110$} & \multirow[b]{2}{*}{.$/ 119$} & \multirow[b]{2}{*}{. / MFF } & مقدار ثابت & \multirow[b]{2}{*}{ زن } \\
\hline $\begin{array}{l}\cdot \cdots 1 \\
* 0\end{array}$ & $r / \uparrow \wedge$ & & & & & مبتنى بر اصول كردن & \\
\hline
\end{tabular}

جدول هـ - نتايج تحليل ركرسيون بين هوش اخلاقى و خرده مقياسهاى آن با معناى زندگى در مردان و زنان. 


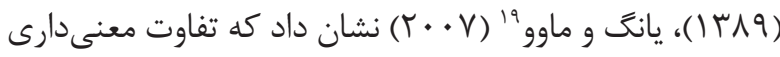

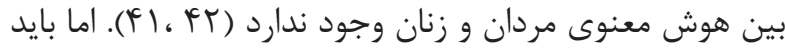

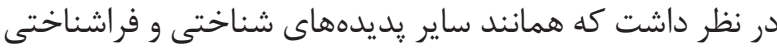

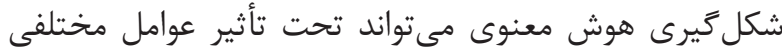

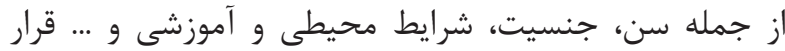

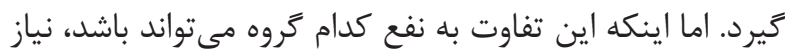

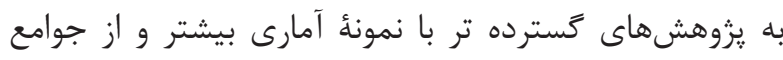
مختلف فرهنكى دارد.

معناى زندكى، مسئلهاى ناظر بر ارزش، غايت و كاركرد زند ركى

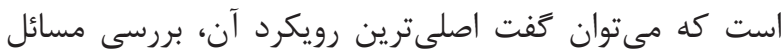

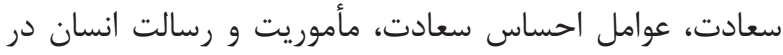

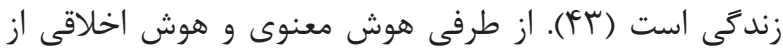

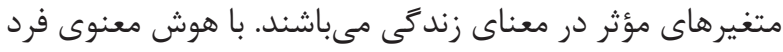

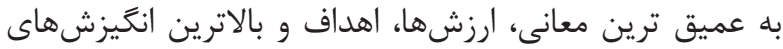

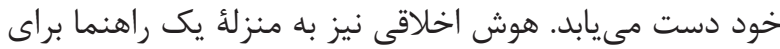

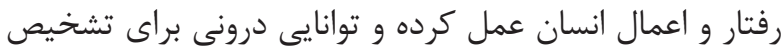

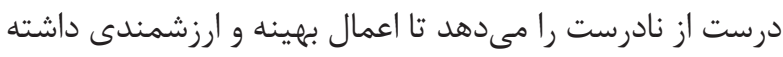

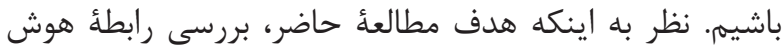

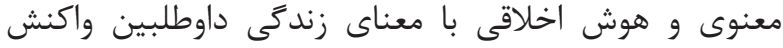
اضطرارى بوده است، يافتهها حاكى از آن آن است كه هوش دئ معندي

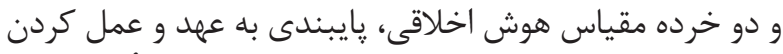

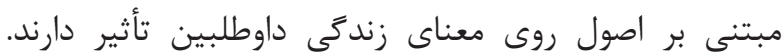

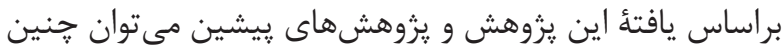

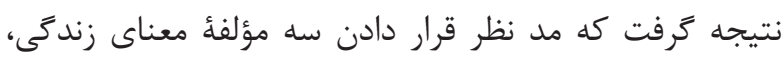

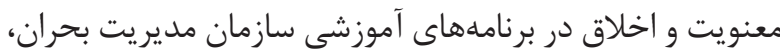

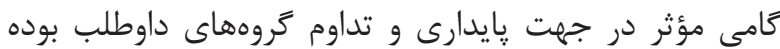

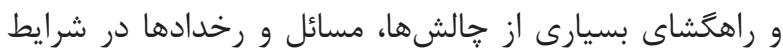

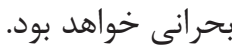

1. Frzadebhtash MR, Agha Babai MT, Mohammad Amini M, Amiri E. local emergency response volunteers design.1st ed. The central study and planning of Tehran city Publication. 2011; p. 7.

2. Hori F. Poetry therapy by poetries of Molana with emphasis on the logotherapy and existentialist. MA Thesis. Azad University of Dezful. 2013.

3. Gol Mohammadiyan M, Frahbakhsh K, Ismailia M. Effectiveness training of moral intelligence. Journal of Counseling and Family Therapy. 2013; 2: 209-32.

4. Parandeh A, Ezadi A, Ebadi A, Ghanbari M. Investigation the spiritual intelligence and organizational commitment in a military hospital nurse managers. JMP. 2011; 2(6): 69-78.

5. Zohar D, Marshal I. Spiritual Capital: Wealth We Can Live by. $1^{\text {st }}$ ed. USA; Berrett-koehler Publishers. 2004.
در مطالعه حاضر مشاهده شد فرضيئ دوم مبنى بر اين كه بـ بين

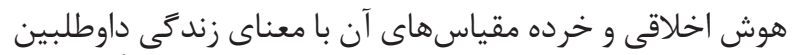

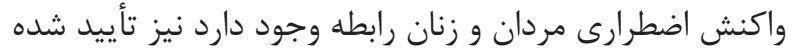

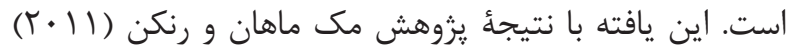

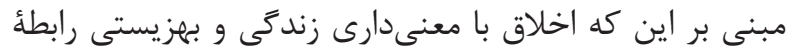

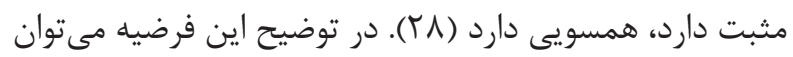

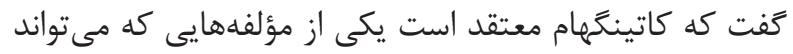

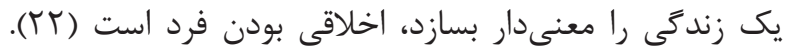

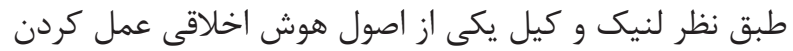

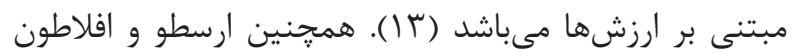

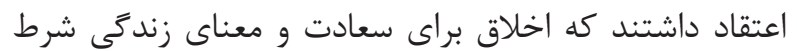

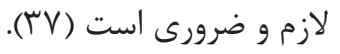

در بررسى فرضيأ سوم نتايج نشان داد تفاوت معنى دارى بين

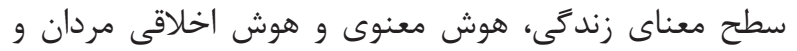

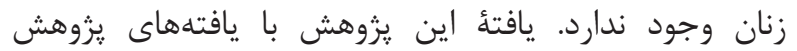

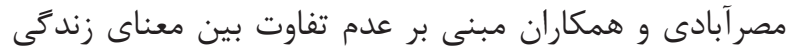

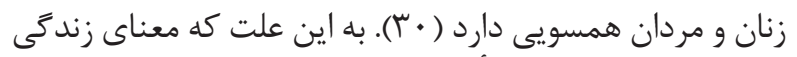

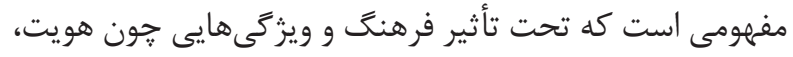

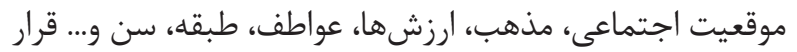

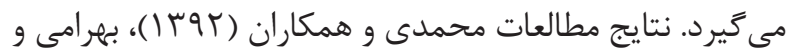

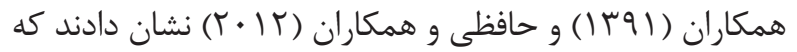

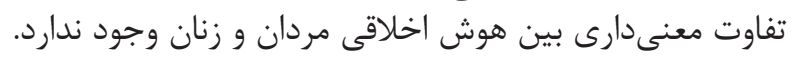

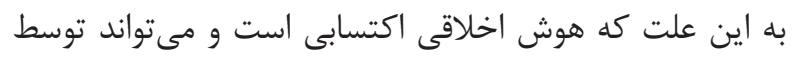

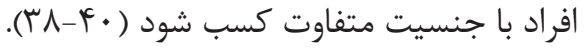

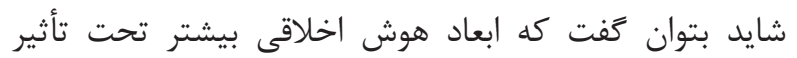

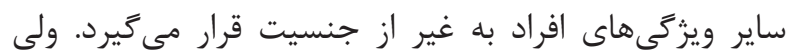

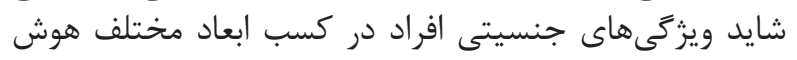

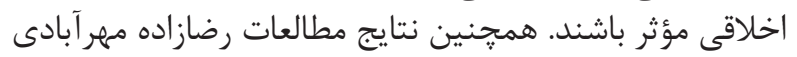

\section{منابع}

6. Golipour s. The relationship between spiritual intelligence and moral intelligence with meaning of life in the volunteers of the emergent reaction. MS Thesis. Garmsar. Azad University. Faculty of Education and Psychology. 2014.

7. Sad Abadi A. The relationship between spiritual intelligence and hopefully with mental health of adolescents in Ray. MS Thesis. Roodhen. Azad University. Roodhen University. Faculty of Education and Psychology. 2011.

8. Ahmadi A, Ahghar G, Abedi MR. The relationship between spiritual intelligence and taking responsibility with life quality. Eur Online J Nat Soc Sci. 2013; 2(3); 391-400.

9. Mahmoudi Kataki Z, Rezaei F, Gorji Y. A social work study on the effect of spiritual intelligence and psychological capital on sense of vitality. Management 


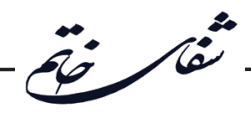

Science Letters. 2013; 3(6): 1559-64.

10. Perrone KM, Webb LK, Wright SL, Jackson ZV, Ksiazak TM. Relationship of Spirituality Intelligence to work and family roles and life satisfaction among gifted adults. AMHC. 2006; 28(3): 253.

11. Clarcen DA. Reflective, ethical and moral constructs in educational leadership preparation: effects on graduates practices. J EDUC ADMIN. 2012; 44(5): 487-508.

12. Ellison L. Moral Reasoning of Chronically Disruptive Pre-adolescent African-American Males in an Urban Elementary School Setting. Doctor of Education (EdD) Thesis. School of Education. St. John Fisher College. 2011.

13. Lennick D. Kiel F. Moral Intelligence: Enhancing Business Performance and Leadership Success. New York: Wharton School. 2008.

14. Flite CA, Harman LB. Code of ethics: Principles for ethical Leadership. Perspect Health Inf Manag. 2013; 10: $1 \mathrm{~d}$.

15. Jafari Nadoshan A, Jafari R, Mir Hosseini RS. Impact of moral intelligence on the self-control of girl student behavior in city Taft. MA Thesis. Tabriz University. 2012.

16. Sohrabi A. The relationship between self-esteem and moral intelligence Employees in junior high school for girls Malayer city. MA Thesis. Malayer University. 2012.

17. Shahrodi Rahvar SH. The relationship between Moral intelligence and commitment in the staff organization specialized medical staff and Medical Equipment. MA Thesis. Tehran. Payame Noor University. 2012.

18. Safar Dokht M. The relationship between moral intelligence of managers with job stress of teachers in high school ofBaboolcity. MAThesis. MazandaranUniversity. Faculty of Humanities and Social Sciences. 2012.

19. Mahmmodi MT, Siyadat SA, Shadan Far F. The relationship of compiler of Moral intelligence and team leadership of training managers of Zahedan Universities. Journal of Educational Sciences. 2012; 19: 107-26.

20. Mokhtari Pour M, Siyadat SA. Comparative study of moral intelligence in view of Quran and Islamic scientists. Journal of Islamic and Psychology Studies. 2010; 3: 97-118.

21. Narvaez D. Moral complexity: The fatal attraction of truthiness and the importance of mature moral functioning. Perspect Psychol Sci. 2010; 5(2): 163-81.

22. Alizamani A, Ghaforiyan M. Compilers of Meaning in life from the perspective of John Cottingham. JPR. 2010; 17: 7-23.
23. Hojjati GH, Alizamani A. Investigation of Meaning, value and limit 'votes Gareth Thompson about Life. Journal of Religion and Culture. 2010; 1: 31-55.

24. Frankl VE. Mans Search for Meaning: An Introduction to Logotherapy. $1^{\text {st }}$ ed. Tehran. Dorsa poblisher. 2011; p. 85.

25. Feldman DB, Snyder CR. Hope and meaningful life: Theoretical and empirical accociations between goaldirected thinking and life meaning. J Soc Clin Psychol. 2005; 24(3): 401-21.

26. Nadi MA, Sajjadian I. Comparsion of Psychological Developments Students in Perception of Meaning of Life. Knowledge and Research in Applied Psychology. 2012; 13(47): 18-28.

27. Joseph C, Lakshmi SS. Spiritual Intelligence at Work. Journal of Soft Skills. 2011; 5(4): 21-30.

28. Mc Mahan EA, Renken MD. Eudaimonic conceptions of well-being, meaning in life, and selfreported well-being: Initial test of a mediational model. J Pers Indiv Differ. 2011; 51(5): 589-94.

29. Steger MF, Frazier P, Oishi S, Kaler M. The meaning in life questionnaire: Assessing the presence of and search for meaning in life. J Couns Psychol. 2006; 53(1): 80-93.

30. Mesrabadi J, Ostovar N, Jafarian S. Discriminative and construct validity of meaning in life questionnaire for Iranian students. Journal of Behavioral Sciences. 2013; 7(1): 83-90.

31. Arasteh HR, Azizi Shamami M, Jafari Rad A, Mohammadi Jvzaniy Z. Investigate the moral intelligence of students. J Culture Strategy. 2010; 10(3): 201-14.

32. Shah Hosseini A. Investigation the Authority and Justifiability spiritual intelligence questionnaire. Garmsar Unit. Azad University. Faculty of Education and Psychology. 2010. [In Persian]

33. Amram Y. The contribution of emotional and spiritual intelligences to effective business leadership. doctoral dissertation. PhD Thesis. Institual of Transpersonal Psychology California, Palo Alto. 2009.

34. King DB. Rethinking claims of spiritual intelligence: A definition, model, and measure. Unpublished Master's Thesis. London Trent University. 2008.

35. Yang K. The spiritual intelligence of Nurses in Taiwan. J nurs Res. 2006; 14: 24-35.

36. Elkins M, Cavendish R. Developing a plan forpediatric spiritual care. Holist Nurs Pract. 2004; 18(4): 179-84.

37. Holmes RL. The basic of ethics of philosophy. $3^{\text {nd }}$ ed. 
Tehran. Ghoghnos Publication. 2010; p.146.

38. Mohammadi S, Nakhaee N, Borhani F, Roshanzadeh M. Moral intelligence in nursing: a cross-sectional study in East of Iran. IJME. 2013; 6(5): 57-66.

39. Bahrami MA, Asami M, Fatehpanah A, Dehghani Tafti A, Ahmadi Tehrani GR. Moral intelligence status of the faculty members and staff of the Shahid Sadoughi University of Medical Sciences of Yazd. IJME. 2012; 5(6): 81-95.

40. Hafizah NH, Zaihairul I, Geshina AMS. Moral Competencies among Malaysian Youth. HEJ. 2012; $1-10:(3) 3$.
41. Reza Zadeh Mehrabadi S. Investigation the relationship between spiritual intelligence and decision-making styles of managers Ferdowsi and Medical Sciences of Mashhad Universities. MA Thesis. Ferdowsi University of Mashhad. Medical Sciences Faculty. 2010.

42. Yang K, Mao X. A study of nurses spiritual intelligence: A cross sectional questionnaire survey. Int J Nurs Stud. 2007; 44: 999-1010.

43. Kasaizadeh M, Ali Zamani AA, Vakili H. Happiness and meaning in Ebne Arabic School. Journal of Comparative Theology. 2012; 8: 43-56. 